\title{
Adaptive Robust Tracking of Nonlinear Systems and with an Application to a Robotic Manipulator
}

\author{
Teh-Lu Liao, Li-Chen Fu, and Chen-Fa Hsu \\ Department of Electrical Engineering \\ National Taiwan University
}

\section{ABSTRAC 1}

Based on the input-output linearization technique and the variable structure control strategy, an adaptive control law is developed so that no prior knowledge of the bounds on the plant uncertainties is required. It is shown that the outputs of the closed loop system asymptotically track the given output trajectories despite the uncertainties, and the tracking errors can be made arbitrarily small. The scheme is then applied to the control of a two degree-of-freedom (DOF) robotic manipulator with unknown payload.

\section{INTRODUCTION}

Consider a class of nonlinear systems in the presence of uncertainties of the following form:

$$
\begin{aligned}
& \dot{x}=f(x)+\Delta f(x)+\sum_{i=0}^{m}\left(g_{i}(x)+\Delta g_{i}(x)\right) u_{i} \\
&=f(x)+\Delta f(x)+(G(x)+\Delta G(x)) u \\
& y_{j}=h_{j}(x), j=1, \ldots, m
\end{aligned}
$$

where $\mathrm{x}(\cdot): \mathbb{R}^{\mathrm{n}} \rightarrow \mathbb{R}^{\mathrm{n}}$ is the system state, $\mathrm{u}(\cdot), \mathrm{y}(\cdot): \mathbb{R}_{+} \rightarrow \mathbb{R}^{\mathrm{m}}$, are system inputs and outputs respectively, $f(\cdot), \mathrm{g}_{\mathbf{i}}(\cdot),: \mathbb{R}^{\mathrm{n}} \rightarrow \mathbb{R}^{\mathrm{n}}$, $i=1, \ldots, m$, are sufficiently smooth vector fields, $h_{i}(\cdot): \mathbb{R}^{n} \rightarrow \mathbb{R}$, $\mathrm{i}=1, \ldots, \mathrm{m}$, are sufficiently smooth scalar functions, and $\Delta \mathrm{f}(\mathrm{x})$ $\Delta \mathrm{g}_{\mathrm{i}}(\mathrm{x}), \quad \mathrm{i}=1, \ldots, \mathrm{m}$ represent uncertainties continuously differentiable with respect to $\mathrm{x}$. The nominal system is then defined as follows: (i.e. with $\Delta \mathrm{f}(\mathrm{x})=0, \Delta \mathrm{G}(\mathrm{x})=0$ in (1.1))

$$
\begin{aligned}
& \dot{x}=f(x)+G(x) u \\
& y_{i}=h_{i}(x) \quad, i=1, \ldots, m
\end{aligned}
$$

There has been a great deal of excitement in recent years over the development of a rather complete theory for explicitly linearizing the input-output response of the nominal system (1.2) using state feedback. This has been explicitly worked out in several papers, like those of $[1,5]$.

Variable structure control $[2-4]$ is a powerful method which can deal with nonlinear systems. It first defines the sliding surface in the error state space and forms a switching feedback control. Also using the bounds of the uncertainties, the fast switching control forces the error state to slide along the surface until it converges and then the tracking is attained.

Based on the input-output linearization and variable structure control strategy, the previous works $[2-4]$ explicitly rely on the knowledge of the bounds on the plant uncertainties, and then the tracking is achieved. Contribution of the present paper can be stated as follows: given desired output trajectories $y_{d}(t) \in \mathbb{R}^{m}$ design an adaptive robust controller, which neither requires the bounds on the uncertainties of the system nor uses discontinuous control, so that the outputs $y=\left(y_{1}, \ldots, y_{m}\right)^{\top}(T$ denotes transpose below) of the closed loop system will attain arbitrarily small tracking errors, i.e. $e=y-y$ are arbitrarily small, disregard the uncertainties $\Delta f$ and $\Delta G$.

\section{ROBUST CONTROL}

The uncertain nonlinear systems under consideration are of the form (1.1) but subject to the following conditions:

(C1) The strong relative degree $\nu_{i}$ of the $i$-th output of the system $(1.2)$ is a finite number, $i=1, \ldots, m$, and $B(x)$ (to be defined shortly in the sequel) is nonsingular for all $x \in \mathbb{R}^{\mathrm{n}}$.

C2) The system (1.2) is exponentially minimum-phase, i.e the equilibrium point 0 of the zero dynamics is exponentially stable. Moreover the zero dynamics satisfy a Lipschitz condition.

Condition ( $\mathrm{C} 1$ ) implies that the nominal system can be linearized by a nonlinear state feedback law of the following form:

$\mathrm{u}=\mathrm{B}(\mathrm{x})^{-1}[-\mathrm{A}(\mathrm{x})+\mathrm{W}]$

where $\mathrm{A}(\mathrm{x})=\left(\mathrm{a}_{1}(\mathrm{x}), \ldots, \mathrm{a}_{\mathrm{m}}(\mathrm{x})\right)^{\top}, \mathrm{B}(\mathrm{x})=\left(\mathrm{b}_{1}(\mathrm{x}), \ldots, \mathrm{b}_{\mathrm{m}}(\mathrm{x})\right)^{\top}$ with $a_{i}(x)=L_{f}^{v} h_{i} h_{i}$ and $b_{i}(x)=\left[L_{g} L_{f}^{v-1} h_{i}, \ldots, L_{g} L_{f}^{v i}{ }^{-1} h_{i}\right]$.

(C3) There exist continuous functions $\mathrm{D}(\cdot): \mathbb{R}^{\mathrm{n}} \rightarrow \mathbb{R}^{\mathrm{m}}, \mathrm{E}(\cdot)$ : $\mathbb{R}^{\mathrm{n}} \rightarrow \mathbb{R}^{\mathrm{mxm}}$ such that the uncertainties $\Delta \mathrm{f}(\mathrm{x})$ and $\Delta \mathrm{G}(\mathrm{x})$ in (1.1) satisfy

$\Delta f(x)=G(x) D(x), \Delta G(x)=G(x) E(x)$.

Now let $y_{d}=\left(y_{d_{1}}, \ldots, y_{d_{m}}\right)^{\top}$ be the desired output trajectories where $y_{d_{i}}{ }^{(j)}, j=0, \ldots, \nu, i=1, \ldots, m$, are bounded signals.. If we denote $e_{i}=y_{i}-y_{d_{i}}, i=1, \ldots, m$, the sliding surface is then defined

$0=s_{i}=e_{i}^{(v-1)}+\sum_{j=1}^{\left(v_{i}-1\right)} \beta_{i j} e^{(v-j-1)}$

where $\beta_{i j}, \mathrm{j}=1, \ldots, \nu,-1, \mathrm{i}=1, \ldots, \mathrm{m}$ are chosen such that the following polynomials:

$$
\hat{H}_{i}(s)=s^{[\nu}{ }_{i}+1 \supset \beta_{i 1} s^{c \nu-2 \jmath}+\cdots \cdot+\beta_{i} \nu_{i}, \quad, \quad i=1, \ldots, \quad m
$$

are Hurwitz. Now let the input $u$ in $(2.1)$ be chosen as follows $\mathrm{u}=\mathrm{B}(\mathrm{x})^{-1}[-\mathrm{A}(\mathrm{x})+\xi(\mathrm{x}, \mathrm{t})-\kappa(\mathrm{x}, \mathrm{t}) \operatorname{sgn}(\mathrm{S})]$

where $\kappa(x, t)$ is a switching state feedback gain to be determined and $\operatorname{sgn}(\mathbf{S})=\left(\operatorname{sgn}\left(\mathrm{s}_{1}\right), \ldots, \operatorname{sgn}\left(\mathrm{s}_{\mathrm{m}}\right)\right)^{\top}$ where $\operatorname{sgn}(\cdot)$ denotes the standard sign function, then $\xi(x, t)=\left(\xi_{1}(x, t), \ldots, \xi_{m}(x, t)\right)^{\top}$ can be shown to satisfy the following:

$\xi_{i}(x, t)=y_{d_{i}}{ }^{(v-1)}-\sum_{j=1}^{\left[v_{i}-1\right)} \beta_{i j} e^{\left(v_{j}-j\right]}$

which is an implementable signal vector.

In the variable structure control theory, the so called sliding condition must be satisfied. So, an additional condition will be required.

(C4) There exist known functions $\psi_{1}(\cdot): \mathbb{R}^{\mathbf{n}} \rightarrow \mathbb{R}_{+}, \psi_{2}(\cdot, \cdot)$ : $\mathbb{R}^{n_{x}} \mathbb{R}_{+} \rightarrow \mathbb{R}_{+}$and $1>\epsilon_{0}>0$ (sufficiently small) such that for all $x$ $\in \mathbb{R}^{\mathrm{n}}$ and $\mathrm{t}>0$

$\left\|\mathrm{B}(\mathrm{x}) \mathrm{E}(\mathrm{x}) \mathrm{B}(\mathrm{x})^{-1}\right\|_{\mathrm{i} \infty} \leq \psi_{1}(\mathrm{x}) \leq 1-\epsilon_{0}$

$\left\|\mathrm{B}(\mathrm{x}) \mathrm{D}(\mathrm{x})+\mathrm{B}(\mathrm{x}) \mathrm{E}(\mathrm{x}) \mathrm{B}(\mathrm{x})^{-1}(-\mathrm{A}(\mathrm{x})+\xi(\mathrm{x}, \mathrm{t}))\right\| \leq \psi_{2}(\mathrm{x}, \mathrm{t})$ 
Lemma 1 [2]: Consider the system (1.1) under conditions (C1)-(C4). Given the desired output trajectories $y_{d}(t) \in \mathbb{R}^{m}$ with the aforementioned properties, the variable structure control law in $(2.5)$ with the switching feedback gain $\kappa(\mathrm{x}, \mathrm{t})$ given as follows:

$$
\kappa(\mathrm{x}, \mathrm{t}) \geq\left(1-\psi_{1}\right)^{-1}\left(\eta+\psi_{2}(\mathrm{x}, \mathrm{t})\right), \quad \eta>0
$$

achieves the asymptotic tracking, i.e. $y(t) \rightarrow y_{d}(t)$ as $t \rightarrow \infty$.

\section{ADAPTIVE ROBUST CONTROI}

In practical application, however, there may be situations in which the bounds (condition $\mathrm{C} 4$ ) are unknown. Therefore, the switching feedback gain $k(\mathrm{x}, \mathrm{t})$ can not be constructed to meet the sliding condition. One possible way to overcome this difficulty is to estimate the gain and update it by some adaptation law so that the sliding condition can be met and the error state reaches the sliding surface and stays on it.

To realize this idea, we first state the following condition which takes the place of the condition (C4).

$\left(\mathrm{C} 4^{\prime}\right)$ There exist known functions $\psi_{1}(\cdot): \mathbb{R}^{\mathrm{n}} \rightarrow \mathbb{R}_{+}^{\ell}, \psi_{2}(\cdot, \cdot)$ : $\mathbb{R}^{\mathrm{m}} \times \mathbb{R}_{+} \rightarrow \mathbb{R}_{+}^{\ell}$ and $1>\epsilon_{0}>0$ (sufficiently small) such that for all $\mathrm{x} \in \mathbb{R}^{\mathrm{n}}$ and $t>0$

$$
\begin{gathered}
\left\|\mathrm{B}(\mathrm{x}) \mathrm{E}(\mathrm{x}) \mathrm{B}(\mathrm{x})^{-1}\right\|_{\mathrm{i}_{\infty}} \leq \mathrm{p}_{1}^{\top} \psi_{1}(\mathrm{x}) \leq 1-\epsilon_{0} \\
\left\|\mathrm{~B}(\mathrm{x}) \mathrm{D}(\mathrm{x})+\mathrm{B}(\mathrm{x}) \mathrm{E}(\mathrm{x}) \mathrm{B}(\mathrm{x})^{-1}(-\mathrm{A}(\mathrm{x})+\xi(\mathrm{x}, \mathrm{t}))\right\| \leq \\
\ell \quad \mathrm{P}_{2}^{\top} \psi_{2}(\mathrm{x}, \mathrm{t}) \\
\ell
\end{gathered}
$$
where $\mathrm{p}_{1} \in \mathbb{R}_{+}^{\ell}$ and $\mathrm{p}_{2} \in \mathbb{R}_{+}^{\ell}$ are unknown parameters. Here, $\mathbb{R}_{+}^{\ell}$

$\left(\mathbb{R}_{+}^{2}\right)$ denotes the $\ell_{1}\left(\ell_{2}\right)$-dimensional space in which each entry is nonnegative. An adaptive control law is described as follows:

$$
\begin{array}{r}
\mathrm{u}=\mathrm{B}(\mathrm{x})^{-1}\left(-\mathrm{A}(\mathrm{x})+\xi(\mathrm{x}, \mathrm{t})-\hat{\kappa}(\mathrm{x}, \mathrm{t}) \operatorname{sat}\left(-\frac{\mathrm{S}}{\phi}\right)\right), \phi>0 \\
\text { and } \quad \hat{\kappa}(\mathrm{x}, \mathrm{t})=\left(1-\hat{\mathrm{P}}_{1}^{\mathrm{T}} \psi_{1}(\mathrm{x})\right)^{-1}\left(\eta+\hat{\mathrm{P}}_{2}^{\mathrm{T}} \psi_{2}(\mathrm{x}, \mathrm{t})\right)
\end{array}
$$

where $\phi$ is the thickness of the boundary layer, $\hat{\mathrm{P}}_{1}$ and $\hat{\mathrm{P}}_{2}$ are estimates of $\mathrm{P}_{1}$ and $\mathrm{P}_{2}$, and $\operatorname{sat}\left(\frac{\mathrm{S}}{\phi}\right)=\left(\operatorname{sat}\left(\frac{\mathrm{s}}{\phi}\right), \ldots ., \operatorname{sat}\left(\frac{\mathrm{s}}{\phi}\right)\right)^{\top}$ with

$$
\operatorname{sat}\left(\frac{\mathrm{s}_{\mathrm{i}}}{\phi}\right)=\left\{\begin{array}{ll}
\operatorname{sign}_{\mathrm{s}}\left(\mathrm{s}_{\mathrm{i}}\right) & \text { if }\left|\mathrm{s}_{\mathrm{i}}\right|>\phi \\
\frac{\mathrm{i}}{\phi} & \text { if }\left|\mathrm{s}_{\mathrm{i}}\right| \leq \phi
\end{array} \quad \mathrm{i}=1,2, \ldots, \mathrm{m} .\right.
$$

The parameter update law is defined as follows:

$$
\begin{aligned}
& \dot{\mathrm{P}_{1}}= \begin{cases}0 & \text { if } \hat{\mathrm{P}}_{1}^{\top} \psi_{1}(\mathrm{x}) \geq 1-\epsilon_{1}, 0<\epsilon_{1}<\epsilon_{0} \\
\Gamma_{1} \psi_{1}(\mathrm{x}) \hat{\kappa}(\mathrm{x})\left\|\mathrm{S}_{0}\right\| & \text { otherwise }\end{cases} \\
& \dot{\hat{\mathrm{P}}}_{2}=\Gamma_{2} \psi_{2}(\mathrm{x}, \mathrm{t})\left\|\mathrm{S}_{0}\right\|, \quad \hat{\mathrm{P}}_{1}(0)=0, \hat{\mathrm{P}}_{2}(0)=0
\end{aligned}
$$

where $\mathrm{S}_{0}=\left(\mathrm{S}_{01}, \ldots, \mathrm{S}_{0 \mathrm{~m}}\right)^{\mathrm{T}}=\mathrm{S}-\phi \operatorname{sat}\left(\frac{\mathrm{S}}{\phi}\right)$ and $\Gamma_{1}=\operatorname{diag}\left(\gamma_{1}, \ldots, \gamma_{\ell}\right)>$ $0, \Gamma_{2}=\operatorname{diag}\left(\gamma_{\ell_{1}+1}, \ldots, \gamma_{\ell_{1}+\ell}\right)>0$ are the adaptation gain matrices. Now we are ready to state our main result.

'I'heorem : Consider the system (1.1) satisfying (C1)-(C3) and $\left(C^{\prime} 4^{\prime}\right)$. Given the desired output trajectories $y_{d}(t) \in \mathbb{R}^{m}$ with the aforementioned properties, the adaptive control law described by $(3.2)(3.3)(3.4)$ will force the tracking error $\mathrm{e}_{\mathrm{y}}(\mathrm{t})=\mathrm{y}(\mathrm{t})-\mathrm{y}_{\mathrm{d}}(\mathrm{t})$ to be as small as desired.
Proof: For the detailed proof, please see [3]

\section{APPLICATION TO A TWO DOF ROBOTIC MANIPULATOR}

A two DOF robotic manipulator studied by [6] is used here to illustrate the efficiency of the adaptive robust control law proposed. The endeffector of the manipulator can be extended or extracted from and rotate about a vertical axis. Let $\left(\mathrm{x}_{1}, \mathrm{x}_{2}\right)$ denote the position of the center of mass, $\mathrm{C}$, of the motional link in polar coordinate so that the equations of motion are given by

$$
\begin{aligned}
& \left(M_{c}+M\right) \ddot{x}_{1}-R\left(x_{1}, M\right) \dot{x}_{2}=v_{1}, \\
& I\left(x_{1}, M\right) \ddot{x}_{2}+2 R\left(x_{1}, M\right) \dot{x}_{12} \dot{x}_{2}=v_{2}
\end{aligned}
$$

where

$\mathrm{R}\left(\mathrm{x}_{1}, \mathrm{M}\right)=\mathrm{M}_{\mathrm{c}} \mathrm{x}_{1}+\mathrm{M}\left(\mathrm{x}_{1}+\mathrm{a}\right), \mathrm{I}\left(\mathrm{x}_{1}, \mathrm{M}\right)=\mathrm{J}_{1}+\mathrm{J}_{2}+\mathrm{M}_{\mathrm{c}} \mathrm{x}_{1}^{2}+\mathrm{M}\left(\mathrm{x}_{1}+\mathrm{a}\right)^{2}$. Suppose the payload mass $M$ is not known. Let $M_{0}$ be the nominal mass and $x_{1}, x_{2}$ be the output trajectories, i.e. $y_{1}=x_{1}$ and $\mathrm{y}_{2}=\mathrm{x}_{2}$. We now define $\mathrm{x}_{3}=\dot{\mathrm{x}}_{1}, \mathrm{x}_{4}=\dot{\mathrm{x}}_{2}$,

$$
\mathrm{u}_{1}=\mathrm{I}\left(\mathrm{x}_{1}, \mathrm{M}_{0}\right)^{-1} \mathrm{v}_{1}, \mathrm{u}_{2}=\mathrm{I}\left(\mathrm{x}_{1}, \mathrm{M}_{0}\right)^{-1} \mathrm{v}_{2}
$$

Then, the state representation of the system (4.1) can be written of the form (1.1).

Condition (C1)-(C4') are seen to hold here, and there exist two unknown parameters $\mathrm{p}_{1}$ and $\mathrm{p}_{2}$.

Simulation Results:

To obtain numerical results, we set $\mathrm{M}_{\mathrm{c}}=100 \mathrm{~kg}, \mathrm{M}_{0}=50 \mathrm{~kg}$, $\mathrm{J}_{1}=100 \quad \mathrm{kgm}^{2}, \quad \mathrm{~J}_{2}=100 \mathrm{kgm}^{2}$, and $\mathrm{a}=1 \mathrm{~m}$. The desired trajectories $\mathrm{y}_{\mathrm{d}}=\left(\mathrm{y}_{\mathrm{d1}}, \mathrm{y}_{\mathrm{d} 2}\right)^{\top}$ are $\mathrm{C}^{2}$ function pair given by

$$
y_{d 1}=1+\cos (0.2 t), \quad y_{d 2}=0.2 \sin (0.2 t)
$$

In Fig. 4.1, the payload mass $M$ is set equal to $100 \mathrm{~kg}$ and the simulation shows that the tracking errors are bounded. Therefore, the controller is shown to be robust with respect to the uncertainties.

[1] Byrnes, C.I. and A. Isidori . "Local stabilization of minimum phase nonlinear system", System Control Lett.10, 9-17, 1987.

[2] Fu, L.C. and T.L. Liao . "Globally stable robust tracking of nonlinear systems using variable structure control and with an application to a robotic manipulator", to appear in IEEE Trans. Automatic Control 1990.

[3] Iyer, A. and S.N. Singh . "Variable structure control of decouplable systems and attitude control of spacecraft in the presence of uncertainty", Proc. American Control Conference, Atlanta, G.A., 2238-2243, 1988.

[4] Liao, T.L., L.C. Fu, and C.F. Hsu, "Adaptive robust tracking of nonlinear systems and with an application to a robotic manipulator", Technical Report, E.E., University of Taiwan, 1990.

[5] Sastry, S.S. and A. Isidori "Adaptive control of linearizable systems", IEEE Trans. Automatic Control 34, 1123-1131, 1989.

[6] Suhada, J. and N.H. Cheng . "Tracking controllers for robot manipulator: a high gain perspective", $A S M E J$. Dynamic System Measurement, and Control 110, 39-46, 1988.
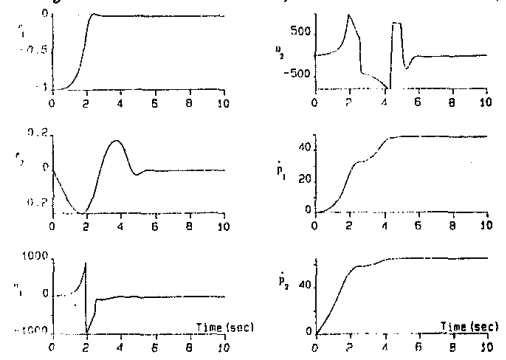

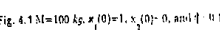

